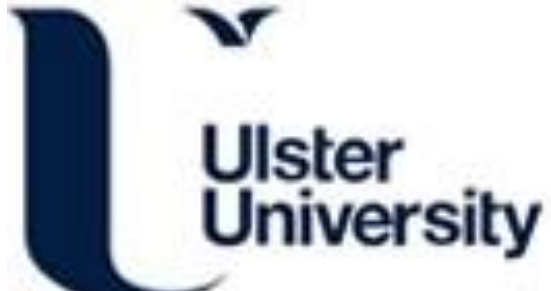

\section{Luminescent Colloidal Silicon Nanocrystals Prepared by Nanoseconds Laser Fragmentation and Laser Ablation in Water}

Svrcek, V., Mariotti, D., Hailstone, R., Fujiwara, H., \& Kondo, M. (2008). Luminescent Colloidal Silicon

Nanocrystals Prepared by Nanoseconds Laser Fragmentation and Laser Ablation in Water. In Unknown Host Publication (pp. 437-442). (MATERIALS RESEARCH SOCIETY SYMPOSIUM PROCEEDINGS).

Link to publication record in Ulster University Research Portal

\section{Published in:}

Unknown Host Publication

Publication Status:

Published (in print/issue): 28/03/2008

\section{Document Version}

Publisher's PDF, also known as Version of record

\section{General rights}

Copyright for the publications made accessible via Ulster University's Research Portal is retained by the author(s) and / or other copyright owners and it is a condition of accessing these publications that users recognise and abide by the legal requirements associated with these rights.

\section{Take down policy}

The Research Portal is Ulster University's institutional repository that provides access to Ulster's research outputs. Every effort has been made to ensure that content in the Research Portal does not infringe any person's rights, or applicable UK laws. If you discover content in the Research Portal that you believe breaches copyright or violates any law, please contact pure-support@ulster.ac.uk. 


\title{
Luminescent Colloidal Silicon Nanocrystals Prepared by Nanoseconds Laser Fragmentation and Laser Ablation in Water
}

\author{
Vladimir Svrcek $^{1}$, Davide Mariotti ${ }^{2}$, Richard Hailstone ${ }^{3}$, Hiroyuki Fujiwara ${ }^{1}$, and Michio Kondo ${ }^{1}$ \\ ${ }^{1}$ Research Center for Photovoltaics, National Institute of Advanced Industrial Science and \\ Technology (AIST), Central 2, Umezono 1-1-1, Tsukuba, 305-8568, Japan \\ ${ }^{2}$ Department of Microelectronic Engineering, Kate Gleason College of Engineering Rochester \\ Institute of Technology, 82 Lomb Memorial Drive, Rochester, NY, NY 14623 \\ ${ }^{3}$ Department of Microelectronic Engineering, Chester F. Carlson Center for Imaging Science, \\ Rochester Institute of Technology, 54 Lomb Memorial Drive, Rochester, NY, NY 14623
}

\begin{abstract}
The surface states of silicon nanocrystals (Si-ncs) considerably affect quantum confinement effects and may determinate final nanocrystals properties. Colloidally dispersed Sincs offer larger freedom for surface modification compared to common plasma enhanced chemical vapor deposition or epitaxial synthesis in a solid matrix. The Si-ncs fabrication and elaboration in water by pulsed laser processing is an attractive alternative for controlling and engineering of nanocrystal surface by environmentally compatible way. We report on the possibility of direct silicon surface ablation and Si-ncs fabrication by nanosecond pulsed laser fragmentation of electrochemically etched Si micrograins and by laser ablation of crystalline silicon target immersed in de-ionized water. Two nanosecond pulsed lasers (Nd:YAG, and excimer $\mathrm{KrF}$ ) are successfully employed to assure fragmentation and ablation in order to produce silicon nanoparticles . Contrary to the fragmentation process, which is more efficient under $\mathrm{Nd}$ :YAG irradiation, the laser ablation by both lasers led to the fabrication of fine and room temperature photoluminescent Si-ncs. The processing that has natural compatibility with the environment and advanced state of fabrication technologies may imply new possibilities and applications.
\end{abstract}

\section{INTRODUCTION}

Silicon nanocrystals (Si-ncs) with grain size of less than $5 \mathrm{~nm}$ exhibit quantum confinement effects and visible photoluminescence (PL) at room temperature and are recognized as one of the potential materials in optoelectronic, bio-imaging, and photovoltaic applications [1, 2]. It is generally accepted that visible PL in Si-ncs originates from surface related recombination that occurs with quantum confinement effect [3]. Fabrication and elaboration of the Si-ncs in liquids may bring some new possibilities for modifying both surface states and quantum confinement [4]. In addition, the host liquid could modify the electron-hole interaction and can be used for optimization of carrier density population under excitation conditions. It has been shown recently that nanosecond pulsed laser ablation in aqueous media is suitable for fabrication of luminescent water soluble Si-ncs [5,6]. Water offers advantageous fabrication conditions that are both environmentally friendly and with unique surface chemistry. The oxide shell that is formed in the fabrication of Si-ncs in water, provides a natural and stable form of surface passivation [5]. The hydrophilic oxide surface may serve as a dielectric shell for further surface functionalization by specific molecular species at the nanoscale level. 
Here, we report on the formation of Si-ncs and surface modification directly in de-ionized water by lasers processing. For the synthesis of Si-ncs two laser processes at room temperature in ambient atmosphere are applied and investigated. The first process involves the fragmentation by nanosecond (ns) laser pulses in water with Si micrograins prepared by electrochemical etching. In the second process, Si-ncs were produced by ns laser ablation of a Si target immersed in deionized water. Two nanosecond pulsed Nd:YAG lasers and an excimer KrF laser are employed to ensure successful fragmentation and ablation and to produce silicon nanoparticles that are dispersed in water. We compare how the preparation with the two nanosecond lasers in aqueous solution at ambient pressure and temperature affects Si-ncs structure, morphology and most importantly luminescence properties.

\section{EXPERIMENT}

The micrograins used for the first process, were prepared by electrochemical etching. For this purpose, silicon wafers (p-type boron doped, $<100>$ ) were etched 2 hours at constant current $1.6 \mathrm{~mA} / \mathrm{cm}^{2}$ in HF:ethanol electrolyte (1:4) and subsequently mechanically pulverized [4, 6]. The micrograins were harvested by sedimentations in ethanol. The $0.01 \mathrm{wt} \% \mathrm{Si}$ micrograins in aqueous solution was then prepared. In order to obtain homogenous dispersion of the hydrophobic Si micrograins [6] in de-ionized water and to ensure micrograins fragmentation, few drops of ethanol have been used to wet the micrograins surface prior to the introduction of water. The colloidal solutions were sonicated for $10 \mathrm{~min}$ and $5 \mathrm{ml}$ of the solution was used for the fragmentation process. Two different nanosecond pulsed lasers (third harmonic of Nd:YAG, Spectra Physics LAB-150-30, 355 nm, 30 Hz, 8 ns and excimer KrF, 245 nm 20 Hz, 10 ns) were employed and compared. The laser irradiation was set at $\sim 6 \mathrm{~mJ} /$ pulse fluence for 2 hours at room temperature and ambient pressure. The laser beam was focused into $1 \mathrm{~mm}$ diameter spot on the liquid surface by a lens. For all cases, the glass containers were closed and rotated during the irradiation.

For the second process, a crystalline silicon wafer with the same parameters as used for micrograins fabrication, was glued at the bottom of the glass container and used as a target for ablation. Nd:YAG and excimer KrF lasers were applied to irradiate onto the target immersed in $10 \mathrm{ml}$ of de-ionized water at room temperature and ambient atmosphere for 2 hours. The laser fluence was set at $5.3 \mathrm{~mJ} /$ pulse. To reveal the PL of the freshly prepared Si-ncs, the solution was kept for several weeks in ambient conditions. A small droplet of the obtained colloidal solutions (prepared by fragmentation or laser ablation) was then deposited onto a copper grid for highresolution transmission electron microscopy (HR-TEM) and scanning electron microscope (SEM) observations. HR-TEM was performed using a microscope with a $200 \mathrm{kV}$ acceleration voltage. Transmission electron diffraction and Raman spectroscopy were employed to perform more localized analyses of Si nanoparticle structure. The PL measurements were performed at room temperature and ambient atmosphere using a fluorophotometer (Shimadzu, RF-5300PC) with excitation by monochromatic light at $300 \mathrm{~nm}$ from a Xe lamp.

\section{LASER FRAGMENTATION}

Silicon surface itself as well as the silicon micrigrains are hydrophobic. Si micrograins can be easily dispersed in almost any organic-based solution. On the other hand, direct dissolution in water is inefficient and micrograins accumulate at the water surface [6]. 
Fragmentation by pulsed laser requires a homogenous dispersion of the Si micrograin in the liquid media. In order to overcome the insolubility of the Si micrograins in water, a small amount of ethanol was used to wet the micrograin surface prior to the introduction of water. Image 1(a) shows a photo of $0.01 \%$ Si grains wetted with ethanol and homogenously dispersed in deionized water. After applying nanosecond pulsed laser irradiation, fragmentation of the micrograins is induced. For both lasers used, it is observed that at low laser fluence $(<1.1$ $\mathrm{mJ} /$ pulse), the solution color changed to dark brown. A prolonged laser irradiation did not induce any additional color change. On the contrary, at higher laser fluence, the irradiation time affected the color of the solutions. In this case, after prolonged irradiation, the solutions became more transparent. Figure 1(b) and Figure 1(c) show solutions after laser ablation for $2 \mathrm{~h}$ at fluence of $5.9 \mathrm{~mJ} /$ pulse by Nd:YAG laser and excimer KrF laser, respectively. The colloid color changed from yellow to mostly transparent after pulsed laser processing. The results show that the fragmentation by Nd:YAG is more efficient and by naked eye the solution became more transparent (Figure 1(b)). This was further confirmed by absorbance measurements. Figure 1(d) shows corresponding absorbance of the solutions before and after laser irradiations. It can be seen that the absorbance of the solution fragmented by excimer KrF laser is higher compared to $\mathrm{Nd}$ :YAG laser. There could be several reasons that justify the different behaviors for the different lasers. One important observation is that the micrograins have quite significant absorption cross-section around $400 \mathrm{~nm}$, which is closer to the $355 \mathrm{~nm}$ wavelength of the $\mathrm{Nd}$ :YAG if compared to the excimer $\mathrm{KrF}$ laser $(249 \mathrm{~nm})$. Therefore the $\mathrm{Nd}$ :YAG laser is expected to show better fragmentation. TEM and SEM images also show that the fragments are smaller after Nd:YAG irradiation with an average diameter of less than $60 \mathrm{~nm}$. In the case of excimer $\mathrm{KrF}$ laser, fragments diameters exceed $100 \mathrm{~nm}$. In both cases the silicon particles maintain the crystalline diamond-like structure. Unfortunately, the particle sizes are quite large and exceed the quantum confinement limit for silicon $(<10 \mathrm{~nm})$. Therefore the visible PL at room temperature in such particles is not observed.
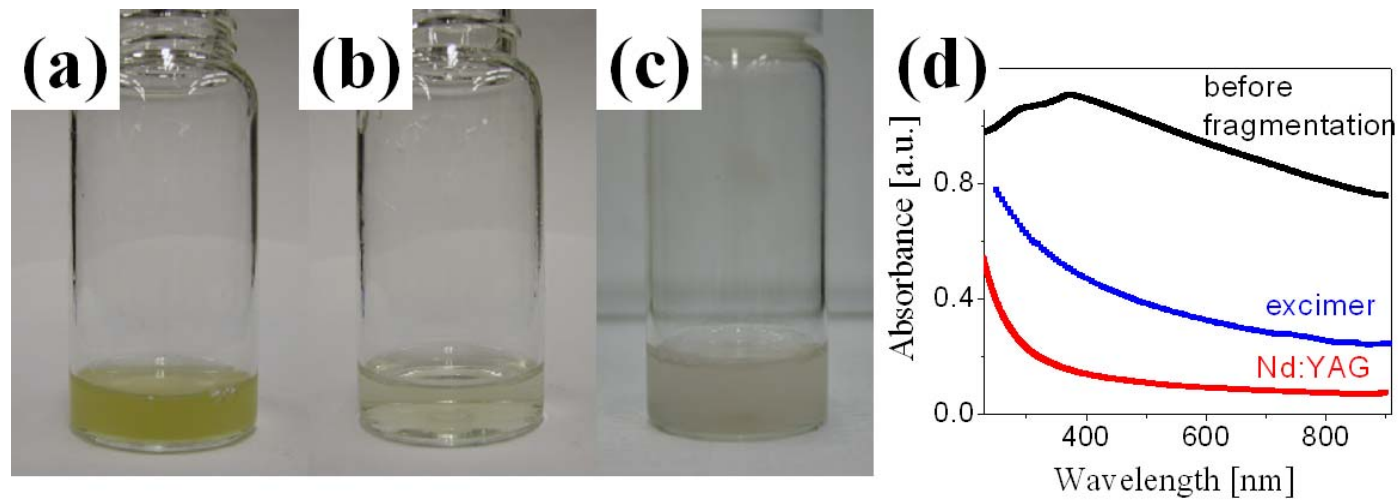

Figure 1. Photos of $0.01 \%$ ethanol wetted Si micro-grains in water (a) before and (b, c) after laser irradiation for 2 hours at a fluence of $\sim 6 \mathrm{~mJ} /$ pulse. Figure (b) and (c) display colloidal suspension after pulsed nanosecond $\mathrm{Nd}$ :Yag and excimer KrF laser irradiations, respectively. (d) Corresponding absorbance of aqueous colloidal solutions before and after laser fragmentation with Nd:Yag (red line) and excimer KrF laser (blue line) are shown.

Several simultaneous processes are responsible for the fragmentation of Si micrograins in both laser irradiation conditions. Similarities can be found with reported results of the 
fragmentation in transparent polymer $[6,7,8]$. For both laser irradiations, a bright spot near the surface level is observed. Optical breakdown of water and explosion sounds can also be heard. These local explosions are due to the collapse of vaporized cavitation bubbles, and of the micrograins fragments that have accidentally diffused under the laser beam. When the Si micrograin absorbs photons from the laser beam a strong electric field is generated causing an electron avalanche and consequent water breakdown. [6, 7]. In the same process, shock waves are also generated. Shockwaves can be accelerated at large distances with the explosion on the silicon micrograins. Despite the large distances traveled by shock waves, they still have sufficient energy to fragment particles [6]. These processes occur only for Si grains homogeneously dispersed in water. It has been verified for both laser conditions that fragmentation did not take place when the grains were not wetted by ethanol and accumulated on the top surface of the aqueous solution[6].

\section{LASER ABLATION}

It has been reported elsewhere that $\mathrm{Si}$-ncs surface oxidation in air improves and stabilizes the luminescent properties of nanocrystals [3]. Water is a natural oxidizing agent and surface modification of silicon directly in water might be an effective way to prepare stable luminescent Si-ncs. It has been reported that by nanosecond pulsed Nd:YAG laser ablation of Si target immersed in water, spherical particles containing Si-ncs can be formed during aging process in deionized water [5, 9]. Similar morphological changes by aging in de-ionized water of Si-ncs are observed for $\mathrm{Si}$-ncs fabricated by excimer $\mathrm{KrF}$ laser as well. Irregular fragments progressively agglomerate and stabilize into separate spheres after few-weeks of aging. Figure 2(a) shows typical TEM image spherical silicon particles after aging in suspension for two weeks. The Sincs were prepared by laser ablation of silicon wafer with laser fluence at $5.3 \mathrm{~mJ} / \mathrm{pulse}$ for 2 hours. We obtained spherical particles with the average size of $40 \mathrm{~nm}$. Further prolonged aging leads to the formation of spheres with the larger diameters [9]. Detailed Raman spectra and HRTEM structural analyses of aged particles prepared by both lasers confirm the presence of Si-ncs with the crystalline silicon diamond-like structure.

Laser heating, adiabatic cooling and final expansion are the main formation processes of $\mathrm{Si}$-ncs by laser ablation in water. A theoretical description of $\mathrm{Si}$-ncs formation was proposed to describe formation of blue luminescent crystalline Si-ncs stabilized in oxidizing liquid by nanosecond pulsed laser ablation [5, 10]. Similar description can be used to interpret the results obtained in this work. Irradiation of nanosecond laser pulses with photon energy $(245 \mathrm{~nm}: \sim 5.06$ $\mathrm{eV}, 355 \mathrm{~nm}: \sim 3.5 \mathrm{eV})$ over the band gap of silicon $\left(E_{g \mathrm{Si}}=1.1 \mathrm{eV}\right)$ leads to a rapid heat generation. Immediately after the absorption of the laser light, a dense cloud of Si atoms spread from the laser forming a typical plasma plume. The confinement of the laser-generated plasma in water significantly enhances Gibbs energy as a dynamical description entity of the Si-ncs formation. In fact, the liberated Gibbs energy of the Si-ncs is kinetic energy subsequently dissipated during the Si-ncs ejection trajectory within the confined laser-induced plume.[8]. In first approximation, the Gibbs energy of Si-ncs formation inside of the water-confined plasma plume as a function of the pressure can be written as follow $[10,11]$

$$
G(P)=\frac{k T}{V} \ln \left(\frac{P(I)}{P_{0}}\right)
$$

where $k$ is the Boltzman constant, and $T$ is the plume temperature. The plasma pressure $P(I)$ in our case is the maximum pressure generated by the lasers in the water. Embryotic Si particles are 
formed together with ejected Si-nc particles. Si atoms rapidly aggregate onto the embryotic particles within the plume until the Si atom density in the surrounding of the forming Si-ncs is drastically reduced. [11]. When the Si-ncs escape from the plasma plume into the water, the growth is suppressed by stacking of silicon-based complexes on the surface. These complexes not only suppress the Si-nc growth but also passivate and stabilize the surface.

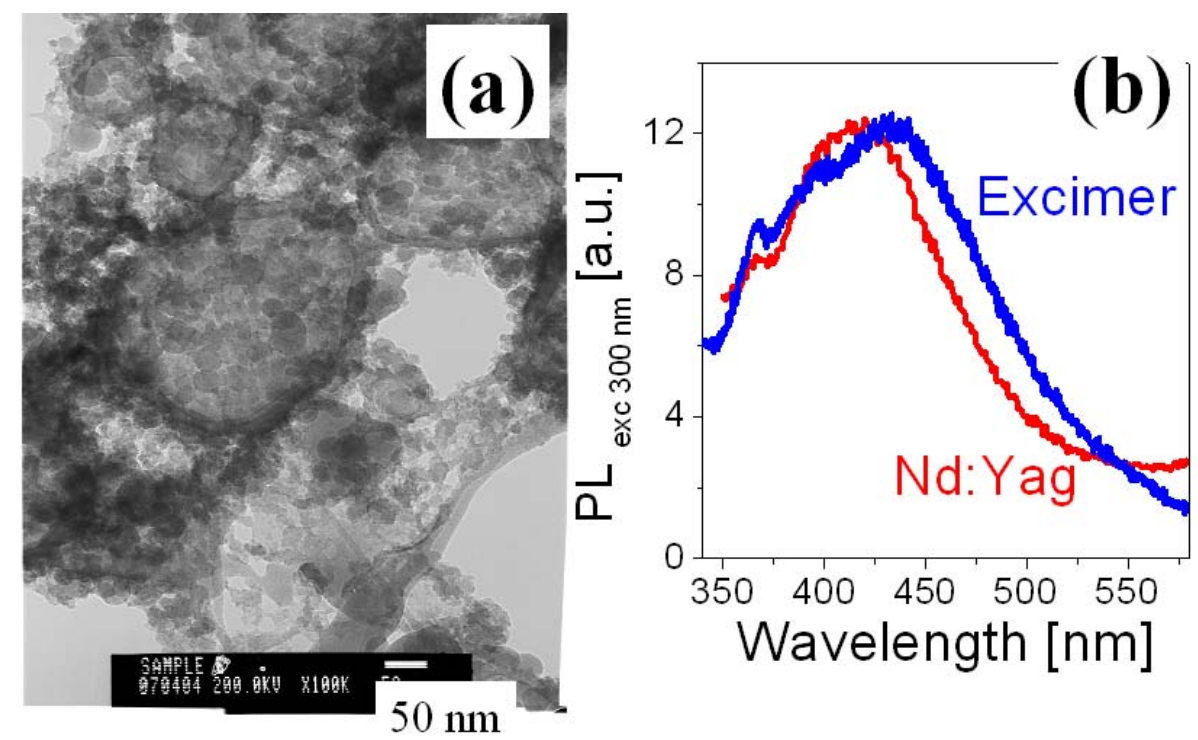

Figure 2. (a) Transmission electron microscopy (TEM) image of silicon nanocrystals (Si-ncs) prepared by nanosecond excimer $\mathrm{KrF}$ laser ablation of silicon wafer in de-ionized water with laser fluence $5.3 \mathrm{~mJ} /$ pulse for 2 hours. (b) Corresponding photoluminescence (PL) spectra from $\mathrm{Si}$-ncs dispersed in water. Blue line represents the PL data of Si-ncs prepared by excimer KrF laser and aged in water for two weeks. The PL spectra of Si-ncs prepared by Nd:YAG laser aged for 4 months are shown for comparisons.

In both cases of laser irradiation, it is observed that as prepared $\mathrm{Si}$-ncs by nanosecond laser ablation dispersed in water showed no visible PL at room temperature. Aging treatment in water for several weeks is necessary to reveal the PL [5]. Corresponding photoluminescence (PL) spectra from Si-ncs dispersed in water prepared by excimer KrF laser and Nd:YAG laser are compared in Figure 2 (b). Rather similar PL spectra with maximum located at $\sim 400 \mathrm{~nm}$ are recorded after aging. The PL intensity of Si-ncs prepared by excimer $\mathrm{KrF}$ laser and aged for two weeks (blue line) is similar to the PL curve obtained for Si-ncs aged for about 4 months and prepared by Nd:YAG laser irradiation (red line). In addition, the KrF excimer laser ablation with shallower absorption produces Si-ncs with red shifted PL peak with maximum centered at 420 $\mathrm{nm}$. The monoxide surface states of Si-ncs together with quantum confinement size effects are responsible for observed photoluminescence at room temperature $[3,5]$. For Nd:YAG laser at least two weeks of aging in de-ionized water is necessary to observe room temperature visible blue PL . Our results indicate that modification and oxide passivation of Si-ncs surface states and defects in oxide layer can effectively shorten by excimer KrF laser ablation. Smaller aggregates of silicon nanocrystals are formed with excimer KrF laser ablation. Then, more Si-ncs are exposed to the water and oxidation may take place during aging more efficiently. This leads to an improvement of the exciton localization and shorter time required for producing visible PL. 


\section{CONCLUSIONS}

Nanosecond laser processing by excimer $\mathrm{KrF}$ and $\mathrm{Nd}-\mathrm{YAG}$ laser was investigated as a cost-effective alternative to modify silicon nanoparticle surface and to synthesize silicon nanocrystals in de-ionized water. Two different lasers have been applied to induce homogeneous fragmentation of micrograins in water and/or to induce laser ablation of silicon wafer immersed in water. It has been shown that the fragmentation by Nd-YAG is more efficient and smaller particles could be produced due to the irradiation wavelength being a closer match to the micrograin absorption cross section. Homogeneous dispersion of Si-ncs is achieved after laser processing due to wetting phenomena of the new-formed particles. However, the particles size exceeds the size required to observe quantum confinement effects and therefore no visible PL is observed at room temperature. This can be overcomed by direct ablation of silicon target immersed in water. Both laser conditions were able to fabricate blue-luminescent ultra-fine Sincs. The aged Si-ncs in de-ionized water exhibit room temperature PL peaked at $400 \mathrm{~nm}$ that can be attributed to quantum confinement effects and oxide-based surface states. During the aging process rather similar morphological and agglomeration phenomena of the Si-ncs into spherical particles were observed. The PL maximum of Si-ncs prepared by excimer KrF is red shifted by $20 \mathrm{~nm}$. It is believed that environmentally friendly and non-toxic silicon nanoparticle fabrication and surface modification might provide a basis for new applications.

\section{REFERENCES}

1. L. T. Canham, Appl. Phys. Lett 57, 1046 (1990).

2. V. Lehmann and U. Gosele, Appl. Phys. Lett. 58, 856 (1991).

3. M. V. Wolkin, J. Jorne, P. M. Fauchet, G. Allan, and C. Delerue, Phys. Rev. Lett. 82, 197 (1999).

4. V. Švrcek, A. Slaoui, and J.-C. Muller, J. Appl. Phys. 95, 3158 (2004).

5. V. Švrcek, T. Sasaki, Y. Shimizu, and N. Koshizaki, Appl. Phys. Lett 89, 213113 (2006).

6. V. Švrcek, T. Sasaki, Y. Shimizu, and N. Koshizaki, Chem. Phys. Lett. 429, 483 (2006).

7. F. Mafune and T. Kondow, Chem. Phys. Lett. 343, 383 (2004).

8. Y. B. Zeldovich and Y. P. Raizer, Physics of Shock Waves and High-Temperature Hydrodynamic Phenomena (Dover Publication, Inc, New York, 2001).

9. V. Švrcek, T. Sasaki, Y. Shimizu, and N. Koshizaki, Journal of Laser Micro/Nanoengineering 2, 15 (2007).

10. A. A. Oraevsky, V. S. Letoshkov, and R. O. Esenafiev, Proceedings of the Workshop"laser ablation: Mechanism and Applications", Pulsed laser ablation of biotissue. review of ablation mechanisms (Springer, Berlin, 1991).

11. V. Švrcek, T. Sasaki, Y. Shimizu and N. Koshizaki, J. Appl. Phys., 103, 023101 (2008). 